\title{
Potential Role of Porcine Reproductive and Respiratory Syndrome Virus Structural Protein GP2 in Apoptosis Inhibition
}

\author{
Sujit Pujhari, ${ }^{1}$ Tayyba T. Baig, ${ }^{2}$ and Alexander N. Zakhartchouk ${ }^{1}$ \\ ${ }^{1}$ International Vaccine Center (VIDO-InterVac), Vaccine and Infectious Disease Organization, University of Saskatchewan, \\ 120 Veterinary Road, Saskatoon, SK, Canada S7N 5E3 \\ ${ }^{2}$ Department of Microbiology and Immunology, University of Saskatchewan, Saskatoon, SK, Canada S7N 5E5
}

Correspondence should be addressed to Alexander N. Zakhartchouk; alex.zak@usask.ca

Received 16 October 2013; Revised 16 December 2013; Accepted 17 December 2013; Published 9 January 2014

Academic Editor: Hanchun Yang

Copyright (C) 2014 Sujit Pujhari et al. This is an open access article distributed under the Creative Commons Attribution License, which permits unrestricted use, distribution, and reproduction in any medium, provided the original work is properly cited.

\begin{abstract}
Porcine reproductive and respiratory syndrome virus (PRRSV) is a serious threat to the pork industry, and its pathogenesis needs further investigations. To study the role of two structural proteins of PRRSV in virus-host cells interactions, two stable cell lines (MARC-2a and MARC-N) expressing GP2 and N proteins, respectively, were established. We induced apoptosis in these cells by treating them with staurosporine and found a significant reduction in the number of apoptotic cells in MARC-2a as compared to MARC-N and MARC-145 cells. In addition, we found significantly higher activities of transcriptional factors (NF- $\kappa$ B and AP- 1 ) in both cell lines as compared to MARC-145 (parent cells). Overall, our data suggest that, although both stable cell lines activate NF- $\kappa$ B and AP-1, GP2 triggers the antiapoptotic process through an intermediate step that needs to be further investigated.
\end{abstract}

\section{Introduction}

Porcine reproductive and respiratory syndrome virus (PRRSV) along with Lactate dehydrogenase-elevating virus, Equine arteritis virus, and Simian hemorrhagic fever virus belongs to the family Arteriviridae of order Nidovirales. It causes one of the most economically significant diseases in the swine industry. In PRRSV, the full-length single stranded genomic RNA is almost $15.4 \mathrm{~kb}$ in length with a $5^{\prime}$ cap and $3^{\prime}$ polyadenylation. It encodes ten ORFs ( $1 \mathrm{a}, 1 \mathrm{~b}, 2 \mathrm{a}, 2 \mathrm{~b} 3,4,5 \mathrm{a}$, $5 b, 6$, and 7), flanked by $5^{\prime}$ and $3^{\prime}$ untranslated regions $[1,2]$. ORF $1 \mathrm{a}$ and $\mathrm{lb}$ constitute the majority of the genome and encode viral nonstructural proteins while the rest codes for structural proteins: GP2, E, GP3, GP4, GP5, 5a, M, and N. A lipid bilayer envelope surrounds the viral RNA embedded within nucleocapsid $(\mathrm{N})$ [3]. Of the structural proteins, GP2, GP3, GP4, and GP5 are glycosylated and present on the viral envelope, along with the nonglycosylated $\mathrm{M}$ and $\mathrm{E}$ proteins. GP5 is known as the major envelope glycoprotein, based on its abundance in the virion, whereas the GP2, GP3, and GP4 are the minor envelope glycoproteins [1]. The $3^{\prime}$-proximal genome part has a compact organization, and most of the genes have overlapping sequences. For instance, ORF $2 b$, encoding the E protein, is partially overlapping ORF 2a that encodes the GP2 protein.

Apoptosis is an important mechanism by which virusinfected cells are eliminated from the host; therefore, many viruses have evolved strategies to prevent or delay apoptosis in order to provide a window of opportunity in which virus replication, assembly, and egress can take place. Interfering with apoptosis may also be required for establishment and/or maintenance of persistent infections.

PRRSV induces apoptosis both in vitro and in vivo [46]. However, it is debatable whether PRRSV induces apoptosis directly (in infected cells) or indirectly (in bystander cells). Lee and Kleiboeker [7] demonstrated that PRRSV induced apoptosis in infected MARC-145 cells through a mitochondria-mediated pathway. In addition, Costers and colleagues [8] showed that both anti- and proapoptotic activities take place in PRRSV-infected MARC-145 cells and macrophages. It appears that, early in infection, PRRSV stimulates antiapoptotic pathways, whereas infected cells die by apoptosis later.

Previous studies demonstrated that PRRSV infection causes an activation of NF- $\kappa$ B and AP- 1 transcription factors [9-11]. Moreover, the ERK signaling pathway is also activated 
in PRRSV-infected cells $[12,13]$, and activation of JNK is required for the virus-induced apoptosis [14]. However, the molecular mechanisms behind these events are poorly understood. Previous studies showed that PRRSV N [11, 15] and nonstructural protein 2 [16] contribute to NF- $\kappa \mathrm{B}$ activation. In the present study, we investigated the role of the PRRSV GP2 and N proteins in apoptosis inhibition and NF- $\kappa \mathrm{B}$ and AP-1 signaling pathways activation using stable cell lines MARC-2a and MARC-N, expressing the GP2 and $\mathrm{N}$ proteins, respectively.

\section{Materials and Methods}

2.1. Cells. MARC-145 (MA-104 clone, African green monkey kidney cell line) cells were maintained in minimal essential medium (Hyclone) supplemented with 10\% fetal bovine serum (FBS), $10 \mathrm{mM}$ HEPES, $10 \mathrm{mM}$ nonessential amino acids, and $100 \mathrm{U}$ of gentamycin.

2.2. Antibodies. NF- $\kappa \mathrm{B}$ rabbit monoclonal antibody (MAb) and c-Jun mouse MAb were purchased from Cell Signaling Technology. Anti-N mouse MAb (SDOW-17) was purchased from Rural Technologies. Fibrillarin mouse MAb was purchased from Santa Cruz. Alpha-tubulin antibody was purchased from Sigma-Aldrich. Cy2-conjugated AffiniPure goat anti-mouse and goat anti-rabbit immunoglobulin G (IgG) were purchased from Jackson ImmunoResearch. Horseradish peroxidase (HRP) conjugated goat anti-mouse IgG and goat anti-rabbit IgG were purchased from Bio-Rad.

2.3. Generation of Stable Cell Lines. Synthesis of the codonoptimized N gene (PRRSV strain VR2332) was ordered from EZBiolab. A 380 bp fragment, amplified by primers (N-FORB-CGggatccATGCCAAATAATAATGGTAA and N-REVB-CGggatccCTAAGCGGATGGAGAAGCAG) was digested with BamHI and inserted into BamHI site of the plasmid pIREShyIA [17] creating pIREShyIA-N.

The pUC57-GP2 plasmid containing a codon-optimized sequence for GP2 gene (PRRSV strain VR2332) was ordered from GenScript and the pIREShyIA-GP2 plasmid for the eukaryotic expression of GP2 protein was constructed as follows: first, a $780 \mathrm{bp}$ fragment was amplified using primers containing BamHI sites (sORF2pUC57ATggatccATGAAGTGGGGCCCCTG and asORF2pUC57ATggatccTCACTGGGAGTTG) and pUC57-GP2 plasmid DNA as a template. Second, this fragment was digested with $B a m \mathrm{HI}$ and inserted into BamHI sites of pIREShyIA vector, creating pIREShyIA-2a. Note, natural GP2 gene (ORF 2a) contains an additional ORF (ORF 2b) encoding E protein. To avoid the expression of $E$ protein, the initiation codon of ORF $2 b$ in the synthetic GP2 gene was mutated from ATG to GTG.

To produce the stable cell lines (MARC-2a and MARC$\mathrm{N})$, subconfluent monolayers of MARC-145 cells were transfected with DNAs of pIREShyIA-2a and pIREShyIA-N, respectively, using Lipofectamine-2000 reagent (Invitrogen) and cell clones were selected by hygromycin B $(300 \mu \mathrm{g} / \mathrm{mL})$.
2.4. NF- $\kappa B$ and AP-1 SEAP Reporter Assay. To monitor the activation of NF- $\kappa \mathrm{B}$ and AP-1 signal transduction pathways, the plasmids ( $\mathrm{pNF} \kappa \mathrm{B}-\mathrm{SEAP}$ or pAP1-SEAP) were purchased from Clontech and were transiently transfected into MARC145, MARC-2a, or MARC-N cells by the use of Lipofectamine LTX (Invitrogen). Briefly, $4 \mu \mathrm{g}$ of each plasmid DNA, $12 \mu \mathrm{L}$ lipofectamine LTX, and $4 \mu \mathrm{L}$ Plus reagent were used to transfect cells, seeded in a well of 6-well plate. Since these transfected plasmids contain the secreted alkaline phosphatase (SEAP) gene as a reporter, culture supernatants were collected $72 \mathrm{~h}$ after transfection, and SEAP activity was detected in by the Great Escape SEAP Chemiluminescence Assay kit (Clontech) using a GloMax 20/20 Luminometer (Promega). The chemiluminescence emitted by a SEAPactivated substrate (CSPD) was measured in relative luminescence units.

2.5. Nuclear and Cytoplasmic Fractionation. MARC-145, MARC-2a, and MARC-N cells were seeded $\left(1 \times 10^{6}\right.$ per well) on 6-well plates. Next day, cells on the plates were washed twice with ice-cold phosphate-buffered saline (PBS) before being scrapped and collected at $1000 \mathrm{rpm}$ for 10 minutes. Cells were resuspended in $450 \mu \mathrm{L}$ buffer A $(100 \mathrm{mM}$ HEPES, pH 7.9, 100 mM KCl, 0.5 mM EDTA, 0.1 mM EGTA, $1 \mathrm{mM}$ dithiothreitol [DTT], and $1 \mathrm{mM}$ phenylmethylsulfonyl fluoride [PMSF]), allowed to swell on ice for 30 minutes, and vortexed for three times/30 s each. Extracts were collected by centrifugation at $14,000 \mathrm{rpm}$ for $10 \mathrm{~min}$ and the supernatant was the cytoplasmic fraction. To remove any residual cytoplasmic extracts contamination, cells were washed using buffer A, followed by PMSF added PBS. The pellet was then resuspended and incubated for 20 minutes in $100 \mu \mathrm{L}$ buffer B (200 mM HEPES, pH 7.9, $500 \mathrm{mM} \mathrm{KCl,} 5.0 \mathrm{mM}$ EDTA, $1.0 \mathrm{mM}$ EGTA, $1 \mathrm{mM}$ DTT, and $1 \mathrm{mM}$ PMSF) and centrifuged at $14,000 \mathrm{rpm}$ for $5 \mathrm{~min}$ at $4^{\circ} \mathrm{C}$. Now, the supernatant was the nuclear fraction. Protein concentrations in both fractions were estimated by Bradford assay, and the purity of the fractions was tested by Western blotting for tubulin and fibrillarin to define the cytoplasmic and nuclear fractions, respectively. Equal amounts of nuclear fractions were analyzed to allow the comparison of protein expression levels in different cells.

2.6. Western Blot Analysis. Denatured protein samples prepared from nuclear and cytoplasmic fractions were resolved by sodium dodecyl sulfate-10\% polyacrylamide gel electrophoresis (SDS-10\% PAGE) and transferred to nitrocellulose membrane. To block nonspecific binding sites on the membrane, it was placed over Tris-buffered saline $(0.1 \mathrm{M}$ Tris [pH 7.6], $0.9 \% \mathrm{NaCl}$ ) containing $0.1 \%$ Tween 20 and $5 \%$ skim milk for $1 \mathrm{~h}$ at room temperature. The membranes were incubated at $4^{\circ} \mathrm{C}$ overnight with appropriate primary antibodies diluted $(1: 1000)$ in $5 \%$ bovine serum albumin (BSA) or $1 \%$ skim milk prepared in $0.1 \%$ TBST as per manufacturer's instructions. HRP-labeled anti-rabbit IgG or anti-mouse IgG detection antibodies diluted (1:2000) in $1 \%$ skim milk prepared in $0.1 \%$ TBST were then added at room temperature for $1 \mathrm{~h}$ and signals were detected with the enhanced chemiluminescence method (Bio-Rad). 


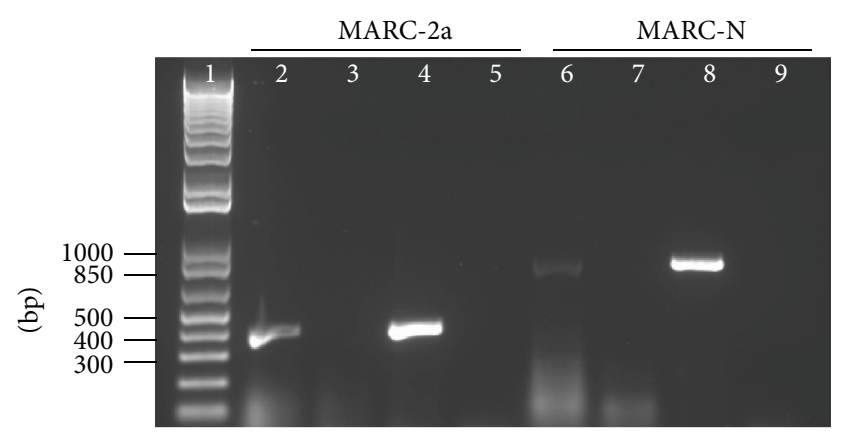

(a)

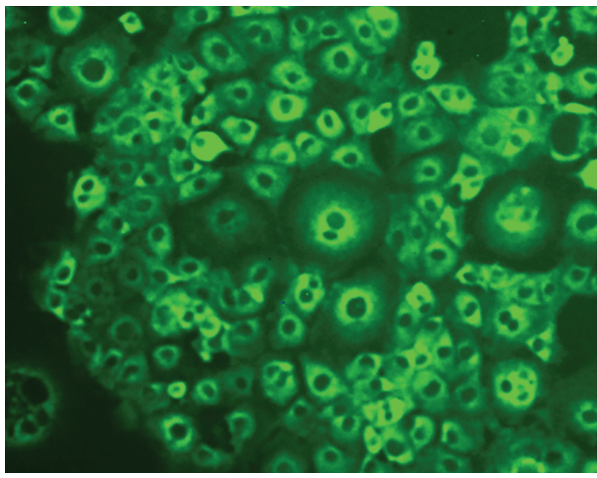

(b)

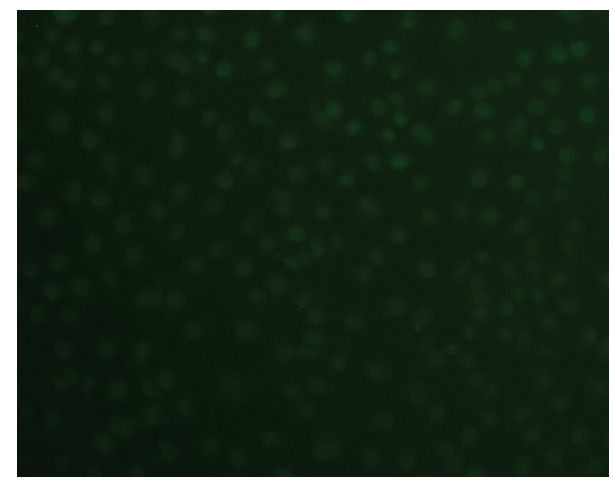

(c)

FIGURE 1: Characterization of GP2 and N expressing stable cell lines. (a) Agarose gel analysis. DNA was extracted from both cell lines and subjected to PCR amplification with primers specific to the $2 \mathrm{a}$ and $\mathrm{N}$ genes. Template amplification with no DNase and no reverse transcription (RT) treatment (lanes 2 and 6); template amplification with DNase treatment and no RT (lanes 3 and 7); template amplification with DNase and RT treatment (lanes 4 and 8); no template controls (lanes 5 and 9). Lane 1 represents the GeneRuler 1 kb Plus DNA Ladder from Fermentas. (b) Immunofluorescence staining of MARC-N cells to show evidence of the $\mathrm{N}$ protein expression. (c) Immunofluorescence staining of MARC-145 parent cells.

The band intensities were measured densitometrically. The band intensities data were normalized with fibrillarin and MARC-145 value was adjusted to 1 for comparison and data were represented as fold changes. For this, the unsaturated bands on the X-ray films were scanned and saved as 8bit grayscale JPEG files and were analyzed by using the public domain software ImageJ from the National Institutes of Health.

2.7. Immunofluorescence Staining. MARC-N and MARC-145 cells at $85-95 \%$ confluency in 2-well Lab-Tek chamber slides were fixed with absolute methanol for $10 \mathrm{~min}$ at $-20^{\circ} \mathrm{C}$. After rehydration with PBS, cells were incubated with anti$\mathrm{N}$ monoclonal antibody SDOW-17 (dilution 1:500) for $1 \mathrm{~h}$ at room temperature. Cells were rinsed three times with PBS and incubated with Cy2-conjugated AffiniPure Goat AntiMouse IgG (dilution 1:200). The cells were examined using a Zeiss Axiovert $200 \mathrm{M}$ inverted fluorescent microscope.

2.8. Assessment of Apoptosis Using Annexin V/PI and Hoechst Staining. A commercially available annexin V apoptosis detection kit (Invitrogen) and flow cytometry were used to determine the annexin V-binding cells. Appropriate cells were grown on $35 \mathrm{~mm}$ disc. Set of wells were treated with
$1 \mu \mathrm{M}$ staurosporine (an apoptotic inducer), for $24 \mathrm{~h}$, keeping another set as an untreated control. After collecting and washing twice with PBS, treated or untreated cells were resuspended in the binding buffer $(500 \mu \mathrm{L})$, followed by the addition of FITC-annexin-V $(5 \mu \mathrm{L})$ PI $(5 \mu \mathrm{L})$ sequentially. The samples were then incubated for $15 \mathrm{~min}$ in the dark at room temperature and subjected to flow cytometric evaluation. The experiment was performed in triplicate and repeated three times.

Nuclear morphology of control and staurosporine treated cells was observed by staining cell nuclei with Hoechst 33342 (Invitrogen). Briefly, cells were incubated with Hoechst 33342 $(10 \mu \mathrm{g} / \mathrm{mL})$ for $15 \mathrm{~min}$ at RT and examined and counted manually (at least 200 per slide) under a fluorescence microscope by using the DAPI filter. Apoptotic cells were characterized by the condensation of chromatin and/or nuclear fragmentation.

2.9. MTT Assay. MARC-145, MARC-2a, or MARC-N cells were split up into 96-well plates at a density of $10^{5}$ cells per well. Next day, staurosporine $(3 \mu \mathrm{M})$ was added. After $24 \mathrm{~h}$, the 3-(4,5-dimethylthiazol-2-yl)-2,5-diphenyl2H-tetrazolium bromide (MTT) assay for mitochondrial activity, an indicator of cell viability, was carried out by adding 


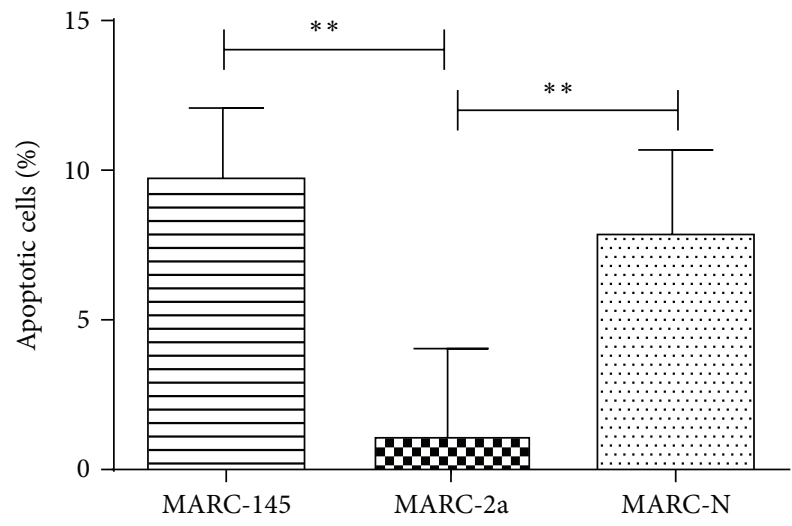

(a)

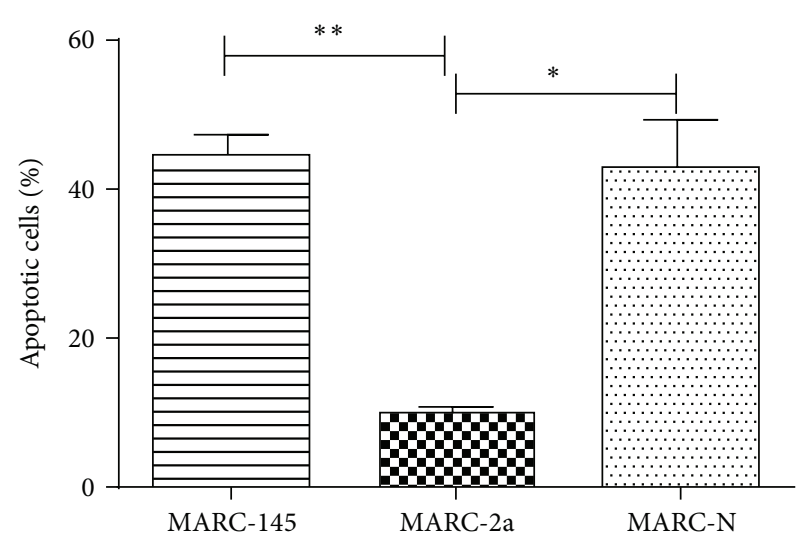

(b)

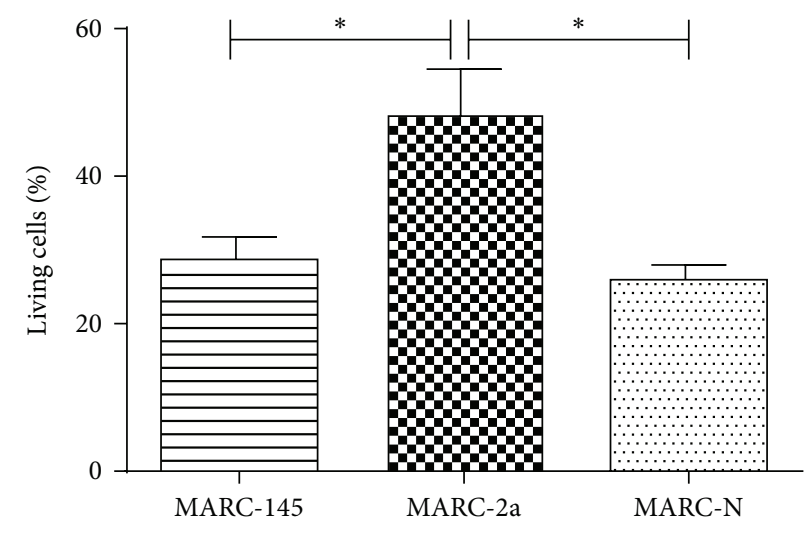

(c)

FIGURE 2: Inhibition of apoptosis in MARC-2a cells. After incubation for $24 \mathrm{~h}$ with $1 \mu \mathrm{M}$ staurosporine, MARC-145, MARC-2a, and MARC-N cells were stained with FITC-annexin V and PI (a) or Hoechst 33342 (b). The percentage of viable cells was determined by MTT assay (c). The data represent the results of three independent experiments in triplicates, and error bars indicate standard deviations of the means. $P<0.05$ $\left({ }^{*}\right) ; P<0.01\left({ }^{* *}\right)$.

$25 \mu \mathrm{L}$ of MTT $(5 \mathrm{mg} / \mathrm{mL})$ to each well and incubating the cells for $2 \mathrm{~h}$ in a $\mathrm{CO}_{2}$ incubator at $37^{\circ} \mathrm{C}$. Finally, $100 \mu \mathrm{L}$ of lysis buffer (20\% SDS in 50\% dimethylformamide, $\mathrm{pH} 4.7$ ) was added, and the cells were further incubated overnight before measuring the optical density at $\mathrm{A}_{595}$ with an ELISA reader. The percentage of living cells was calculated using the following formula: $\left(\mathrm{A}_{595}\right.$ treated cells $) /\left(\mathrm{A}_{595}\right.$ nontreated cells) $\times 100$. The assay was performed in triplicate, and the experiment was repeated three times.

2.10. Statistical Analysis. All data were analyzed using the GraphPad Prism (Version 5.03) software. Differences among all groups were examined using the one-way ANOVA followed by Tukeys test. Differences were considered significant if $P<0.05$.

\section{Results}

3.1. Construction of MARC-2a and MARC-N Cell Lines. To analyze molecular and pathological roles of GP2 and N proteins, we constructed two stable cell lines MARC-2a and MARC-N, expressing PRRSV GP2 and N proteins, respectively. The parent cell line used for this purpose was MARC145 cell line because it supports infection and propagation of PRRSV.

The plasmids pIREShyIA-2a and pIREShyIA-N contained the gene sequences for GP2 and N, respectively, under the control of the human cytomegalovirus promoter, and they also contained the hygromycin B phosphotransferase gene as a selectable marker fused to the internal ribosome entry site (IRES) sequence at the $5^{\prime}$ end. The IRES permits the translation of two open reading frames from one mRNA. MARC-145 cells were transfected with these engineered plasmids and grown under the selection pressure of hygromycin B. Hygromycin-resistant cell clones were further expanded and maintained in the presence of hygromycin B for several days. Cells from each of the maintained clones (MARC-2a and MARC-N) were used for the extraction of total nucleic acids. PCR analysis with the primers specific to the $2 \mathrm{a}$ and N genes revealed the amplified products of $380 \mathrm{bp}$ and $780 \mathrm{bp}$, 


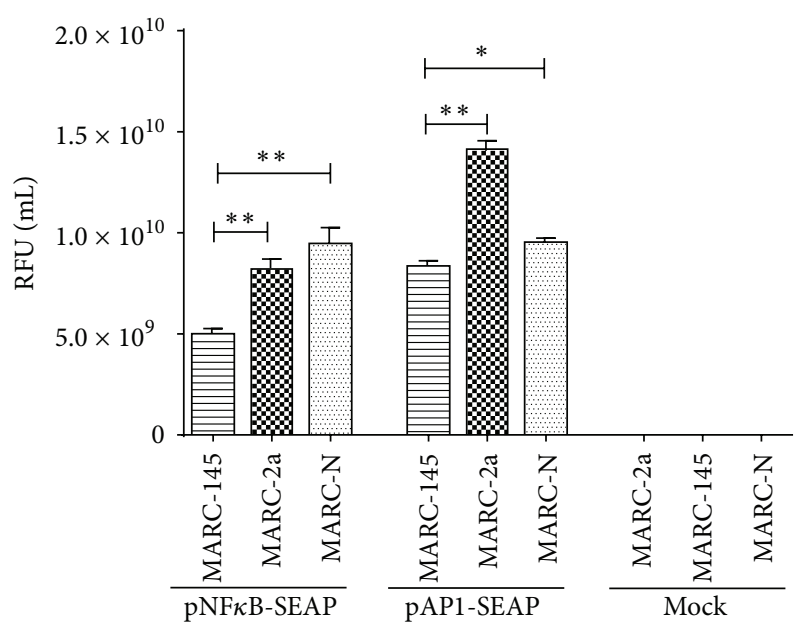

FIgURE 3: NF- $\kappa$ B and AP-1 activity in MARC-145, MARC-2a, and MARC-N cells. Cells were transfected with the secreted alkaline phosphatase (SEAP) reporter vectors: $\mathrm{pNF} \kappa \mathrm{B}-\mathrm{SEAP}$ and $\mathrm{pAP1}$ SEAP. The culture supernatants were collected and analyzed for SEAP activity and represented as relative fluorescence unit (RFU) per $\mathrm{mL}$. The data represent the results of three independent experiments in triplicates, and error bars indicate standard deviations of the means. $P<0.05\left({ }^{*}\right) ; P<0.01\left({ }^{* *}\right)$.

respectively (Figure 1(a), lanes 2 and 6). To confirm the presence of mRNA corresponding to these genes in the cells, the nucleic acids were first treated with DNase, and then an aliquot of each reaction was used as a template for cDNA synthesis followed by PCR amplification (Figure 1(a), lanes 4 and 8). Another aliquot from each reaction was used directly as a template for PCR (Figure 1(a), lanes 3 and 7) to rule out the possibility that the amplified products, shown in lanes 4 and 8 , were from DNA and not from mRNA.

Following the confirmation of mRNA expression, the expression of $\mathrm{N}$-gene at the protein level was evidenced by immunofluorescence staining only in MARC-N cells (Figure 1(b)) and not in parent MARC-145 cells (Figure 1(c)). For $2 \mathrm{a}$ gene, we could not show protein expression due to the unavailability of the GP2-specific antibody.

3.2. Evaluation of Apoptosis Inhibition. To study apoptosis inhibition in stable cell lines, MARC-145, MARC-2a, and MARC-N cells were stained with annexin V-FITC and PI and analyzed by FACS to detect and quantitatively determine the percentage of dead, viable, apoptotic, and necrotic cells. To induce apoptosis, the cells were treated with staurosporine and analyzed at $24 \mathrm{~h}$ after treatment (Figure $2(\mathrm{a})$ ). We found a significantly low percentage of MARC-2a cells $(1.5 \%)$ that were positive for annexin V-FITC (apoptotic) in comparison to MARC-145 (9.5\%) and MARC-N $(8.3 \%)$ cells $(P<0.05)$.

For further verification of these findings, staurosporine treated cells were stained with Hoechst. This nuclear stain allowed discriminating normal live cells (blue homogenate fluorescence) from apoptotic cells with fragmented nucleus and condensed chromatin material (brighter granulated blue color fluorescence). In agreement with the annexin $\mathrm{V}$ staining, MARC-2a cells had significantly lower percentage of apoptotic cells (Figure 2(b)).

Finally, after treatment with staurosporine, the percentage of viable MARC-2a cells was higher than the percentage of living cells in MARC-145 and MARC-N cell lines (Figure 2(c)).

Taken together, these data indicate that MARC-2a cells are more resistant to programmed cell death than to parent MARC-145 cells or MARC-N cells.

3.3. NF- $\kappa B$ and AP-1 Activation. NF- $\kappa \mathrm{B}$ and AP-1 activation in MARC-2a and MARC-N cells was confirmed using the SEAP reporter gene system. The principal behind this assay is the following. If the $\mathrm{NF}-\kappa \mathrm{B}$ or $\mathrm{AP}-1$ signal transduction pathway is induced, endogenous NF- $\kappa \mathrm{B} / \mathrm{AP}-1$ binds to the $\mathrm{k} / \mathrm{Ap}$ enhancer element, located in the promoter region of the $\mathrm{pNF} \kappa \mathrm{B}-\mathrm{SEAP}$ or $\mathrm{pAP} 1-\mathrm{SEAP}$ vector, thus activating the transcription of the SEAP reporter gene.

As shown in Figure 3, MARC-2a and MARC-N cells had significantly increased levels of SEAP than MARC-145 cells, which indicate the NF- $\kappa \mathrm{B}$ and AP-1 activation in these cells. Untransfected cells did not show any SEAP activity.

Additionally, these data were confirmed by Western blot analysis (Figure 4). For biological functions, transcription factors have to go to the nucleus. Thus, we have analyzed the nuclear fractions of MARC-145, MARC-2a and MARC$\mathrm{N}$ cells for the presence of NF- $\kappa \mathrm{B}$ and $\mathrm{c}$-Jun (part of AP1) proteins. Fibrillarin, the nucleolar protein, was used as the loading control. From ImageJ analysis, there was found a 6-fold and 8.5-fold increase in the expression of NF- $\kappa \mathrm{B}$ in MARC-2a, and MARC-N cells, respectively, as compared to MARC-145 cells (Figure 4(a)). In case of AP-1, the fold increase was 4.5 and 4 , respectively (Figure $4(\mathrm{~b})$ ).

\section{Discussion}

Many viruses, including PRRSV, utilize strategies of delaying the process of apoptosis for their benefit. Apoptosis can be triggered by a variety of stimuli including death receptors on the cell surface (extrinsic pathway) and intracellular signals (intrinsic pathway). Staurosporine, a strong inhibitor of protein kinases [18], triggers intrinsic pathway which results in the release of cytochrome $\mathrm{c}$ from mitochondria, formation of the apoptosome (cytochrome c, APAF-1, and caspase-9), and activation of caspase-9. Both the extrinsic and the intrinsic processes congregate at the activation of the downstream effector caspases, which are responsible for inducing the morphological changes observed in an apoptotic cell. The apoptotic events are regulated by the interplay of pro- and antiapoptotic proteins which are members of the Bcl-2 family [19].

Many viruses encode homologue of Bcl-2 proteins that can prevent apoptosis, thus helping the viruses to complete their life cycle in the host cells [20]. For instance, human adenovirus E1B $19 \mathrm{~K}$ protein inhibits apoptosis by forming heterodimers with a variety of proapoptotic proteins of the 

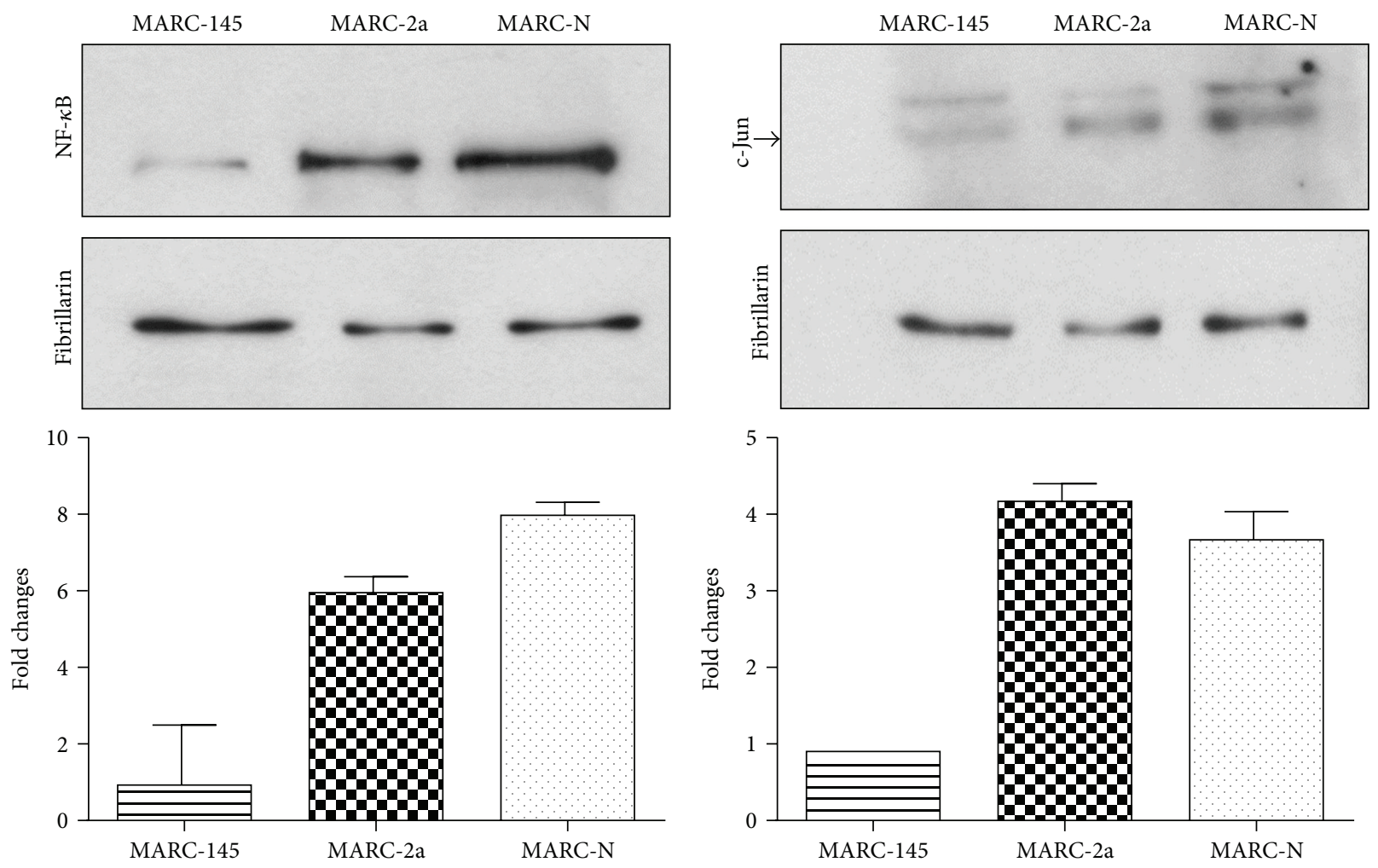

(a)

(b)

FIGURE 4: Western blot analysis of NF- $\kappa$ B and AP-1 activation. Equal amounts of nuclear protein fractions were separated on $10 \%$ SDS-PAGE. Cytoplasmic fraction contamination was evaluated by $\alpha$-tubulin antibody (data not shown). Fibrillarin antibody was used as a nuclear marker and also as a loading control. Fold changes in protein amounts are plotted. Western blot with (a) NF- $\kappa$ B rabbit monoclonal antibody and (b) c-Jun mouse monoclonal antibody. Arrow shows the position of the $48 \mathrm{kDa}$ band corresponding to the phosphorylated form of the c-Jun protein.

Bcl-2 family [21]. However, our attempts to detect interactions between GP2 and proapoptotic members of the Bcl-2 family were not successful (data not shown).

On the other hand, the cell survival in many viral infections is regulated by the NF- $\kappa \mathrm{B}$ activity. For instance, expression of the human hepatitis $\mathrm{C}$ core protein activates the NF- $\kappa$ B pathway which results in an antiapoptotic activity [22]. Also, interferon (IFN) signaling activates the NF- $\kappa \mathrm{B}$ that integrates into the IFN receptor pathways and promotes cell survival [23]. It is also proved that IL-15 has an antiapoptotic effect $[24,25]$. The antiapoptotic property of IL-15 and its regulation by NF- $\kappa$ B signaling in PRRSV infection hinted a possible association with the cell survival strategy of PRRSV. On the other hand, AP-1 has an evolutionary origin from the avian sarcoma virus with cell proliferation and differentiation property that support its role in cell survival signaling [26].

\section{Conclusions}

The main goal of our research is to understand the role of the PRRSV structural proteins in the viral pathogenesis. In the current study, we have explored the role of PRRSV GP2 and $\mathrm{N}$ proteins in activation of the NF- $\kappa \mathrm{B}$ and AP-1 signaling pathways. The role of the $\mathrm{N}$ protein in activation of NF- $\kappa \mathrm{B}$ has been established earlier, whereas activation of cell genes transcription by GP2 has never been previously reported. To our best knowledge, this study is the first report, describing genes transcription activation function of GP2 protein. We have also demonstrated that $\mathrm{N}$ activates the AP-1 pathway in addition to NF- $\kappa \mathrm{B}$, which is a novel finding too.

The GP2 protein likely plays a role in apoptotic inhibition by PRRSV. Possibly, the transcription factors activated by GP2 protein are involved in this activity by activation of antiapoptotic and suppression of proapoptotic genes expression. However, the mechanism of this GP2 function needs to be further investigated.

\section{Conflict of Interests}

The authors declare that there is no conflict of interests regarding the publication of this paper.

\section{Acknowledgments}

The authors thank Mrs. Liubov Lobanova for the technical help. This work was funded in part by the Saskatchewan 
Ministry of Agriculture (Agriculture Development Fund). This paper was published with the permission of the Director of VIDO-InterVac, journal series no. 685.

\section{References}

[1] N. Music and C. A. Gagnon, "The role of porcine reproductive and respiratory syndrome (PRRS) virus structural and non-structural proteins in virus pathogenesis," Animal Health Research Reviews, vol. 11, no. 2, pp. 135-163, 2010.

[2] E. J. Snijder, M. Kikkert, and Y. Fang, "Arterivirus molecular biology and pathogenesis," The Journal of General Virology, vol. 94, part 10, pp. 2141-2163, 2013.

[3] T. Dokland, “The structural biology of PRRSV," Virus Research, vol. 154, no. 1-2, pp. 86-97, 2010.

[4] L. C. Miller and J. M. Fox, "Apoptosis and porcine reproductive and respiratory syndrome virus," Veterinary Immunology and Immunopathology, vol. 102, no. 3, pp. 131-142, 2004.

[5] T. S. Kim, D. A. Benfield, and R. R. R. Rowland, "Porcine reproductive and respiratory syndrome virus-induced cell death exhibits features consistent with a nontypical form of apoptosis," Virus Research, vol. 85, no. 2, pp. 133-140, 2002.

[6] J.-H. Sur, A. R. Doster, and F. A. Osorio, "Apoptosis induced in vivo during acute infection by porcine reproductive and respiratory syndrome virus," Veterinary Pathology, vol. 35, no. 6, pp. 506-514, 1998.

[7] S.-M. Lee and S. B. Kleiboeker, "Porcine reproductive and respiratory syndrome virus induces apoptosis through a mitochondria-mediated pathway," Virology, vol. 365, no. 2, pp. 419-434, 2007.

[8] S. Costers, D. J. Lefebvre, P. L. Delputte, and H. J. Nauwynck, "Porcine reproductive and respiratory syndrome virus modulates apoptosis during replication in alveolar macrophages," Archives of Virology, vol. 153, no. 8, pp. 1453-1465, 2008.

[9] S.-M. Lee and S. B. Kleiboeker, "Porcine arterivirus activates the NF- $\kappa$ B pathway through I $\kappa$ B degradation," Virology, vol. 342, no. 1, pp. 47-59, 2005.

[10] R. Luo, S. Xiao, Y. Jiang et al., "Porcine reproductive and respiratory syndrome virus (PRRSV) suppresses interferon- $\beta$ production by interfering with the RIG-I signaling pathway," Molecular Immunology, vol. 45, no. 10, pp. 2839-2846, 2008.

[11] Y. Fu, R. Quan, H. Zhang, J. Hou, J. Tang, and W. H. Feng, "Porcine reproductive and respiratory syndrome virus induces interleukin-15 through the NF- $\kappa$ B signaling pathway," Journal of Virology, vol. 86, no. 14, pp. 7625-7636, 2012.

[12] Y. J. Lee and C. Lee, "Porcine reproductive and respiratory syndrome virus replication is suppressed by inhibition of the extracellular signal-regulated kinase (ERK) signaling pathway," Virus Research, vol. 152, no. 1-2, pp. 50-58, 2010.

[13] Y. Wang, R. Luo, L. Fang et al., "Porcine reproductive and respiratory syndrome virus (PRRSV) infection activates chemokine RANTES in MARC-145 cells," Molecular Immunology, vol. 48, no. 4, pp. 586-591, 2011.

[14] S. Yin, Y. Huo, Y. Dong et al., "Activation of c-Jun NH(2)terminal kinase is required for porcine reproductive and respiratory syndrome virus-induced apoptosis but not for virus replication," Virus Research, vol. 166, no. 1-2, pp. 103-108, 2012.

[15] R. Luo, L. Fang, Y. Jiang et al., "Activation of NF- $\kappa$ B by nucleocapsid protein of the porcine reproductive and respiratory syndrome virus," Virus Genes, vol. 42, no. 1, pp. 76-81, 2011.
[16] Y. Fang, L. Fang, Y. Wang et al., "Porcine reproductive and respiratory syndrome virus nonstructural protein 2 contributes to NF- $\kappa$ B activation," Virology Journal, vol. 9, article 83, 2012.

[17] A. Zakhartchouk, Y. Zhou, and S. K. Tikoo, "A recombinant E1deleted porcine adenovirus-3 as an expression vector," Virology, vol. 313, no. 2, pp. 377-386, 2003.

[18] V. Sanchez, M. Lucas, A. Sanz, and R. Goberna, "Decreased protein kinase $\mathrm{C}$ activity is associated with programmed cell death (apoptosis) in freshly isolated rat hepatocytes," Bioscience Reports, vol. 12, no. 3, pp. 199-206, 1992.

[19] M. E. Soriano and L. Scorrano, "The interplay between BCL2 family proteins and mitochondrial morphology in the regulation of apoptosis," Advances in Experimental Medicine and Biology, vol. 687, pp. 97-114, 2010.

[20] B. J. Thomson, "Viruses and apoptosis," International Journal of Experimental Pathology, vol. 82, no. 2, pp. 65-76, 2001.

[21] D. Perez and E. White, "TNF- $\alpha$ signals apoptosis through a biddependent conformational change in Bax that is inhibited by E1B 19K," Molecular Cell, vol. 6, no. 1, pp. 53-63, 2000.

[22] Y. M. Chung, K. J. Park, S. Y. Choi, S. B. Hwang, and S. Y. Lee, "Hepatitis $\mathrm{C}$ virus core protein potentiates TNF- $\alpha$-induced NF- $\kappa$ B activation through TRAF2-IKK $\beta$-dependent pathway," Biochemical and Biophysical Research Communications, vol. 284, no. 1, pp. 15-19, 2001.

[23] C. H. Yang, A. Murti, S. R. Pfeffer, L. Basu, J. G. Kim, and L. M. Pfeffer, "IFN $\alpha / \beta$ promotes cell survival by activating NF- $\kappa \mathrm{B}$," Proceedings of the National Academy of Sciences of the United States of America, vol. 97, no. 25, pp. 13631-13636, 2000.

[24] S. Bulfone-Paus, D. Ungureanu, T. Pohl et al., "Interleukin-15 protects from lethal apoptosis in vivo," Nature Medicine, vol. 3, no. 10, pp. 1124-1128, 1997.

[25] S. Inoue, J. Unsinger, C. G. Davis et al., "IL-15 prevents apoptosis, reverses innate and adaptive immune dysfunction, and improves survival in sepsis," The Journal of Immunology, vol. 184, no. 3, pp. 1401-1409, 2010.

[26] P. K. Vogt, "Retroviral oncogenes: a historical primer," Nature Reviews Cancer, vol. 12, no. 9, pp. 639-648, 2012. 

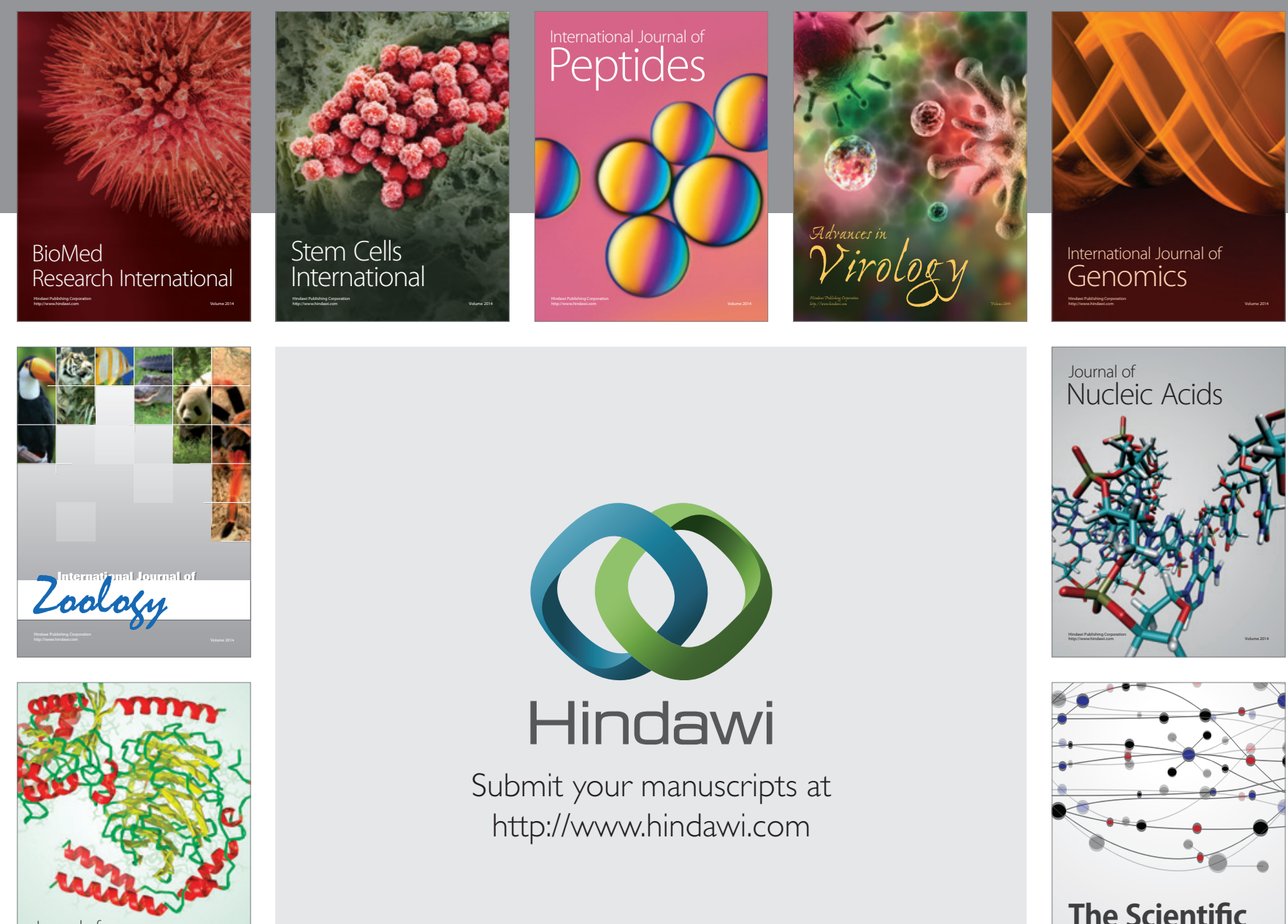

Submit your manuscripts at

http://www.hindawi.com

Journal of
Signal Transduction
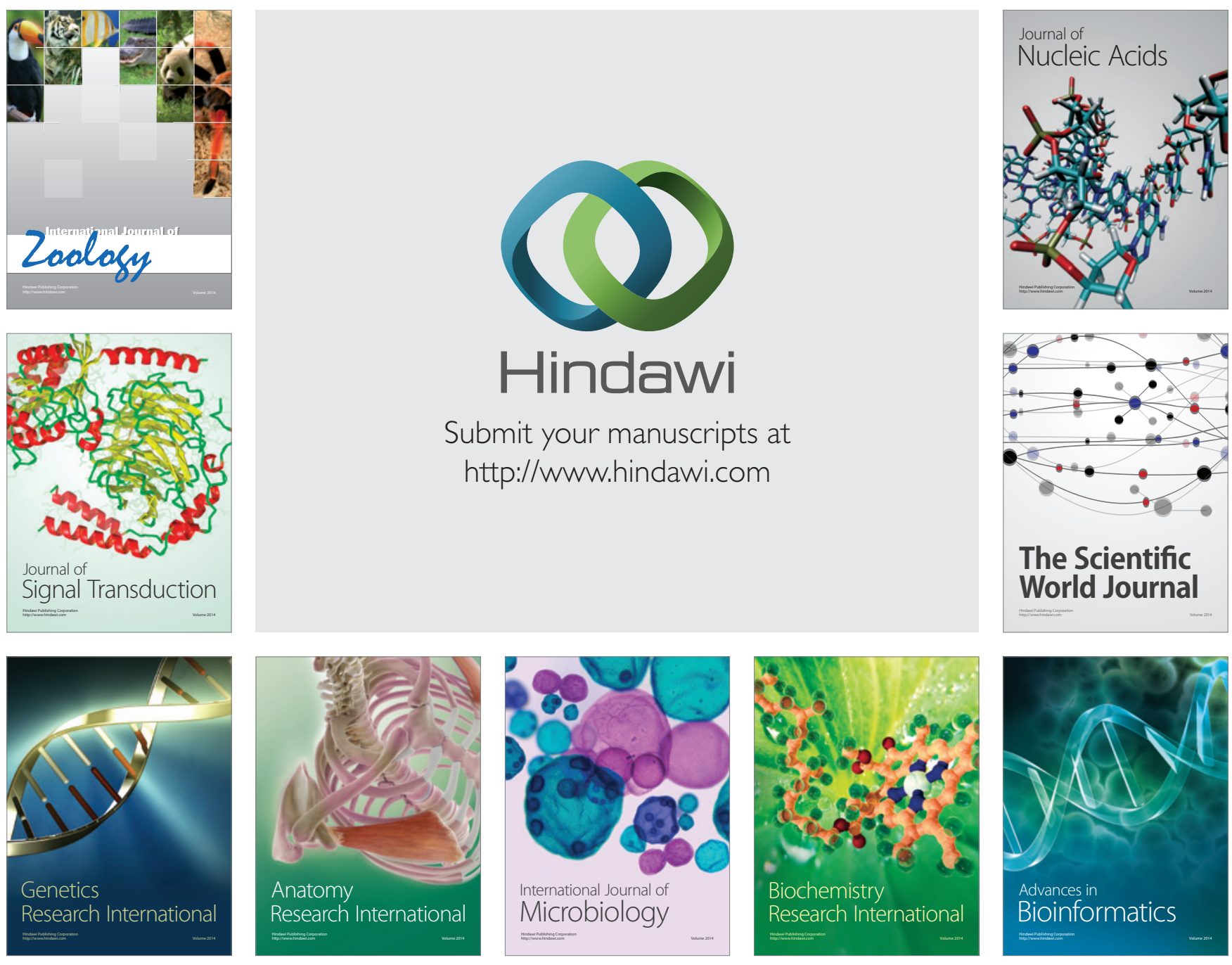

The Scientific World Journal
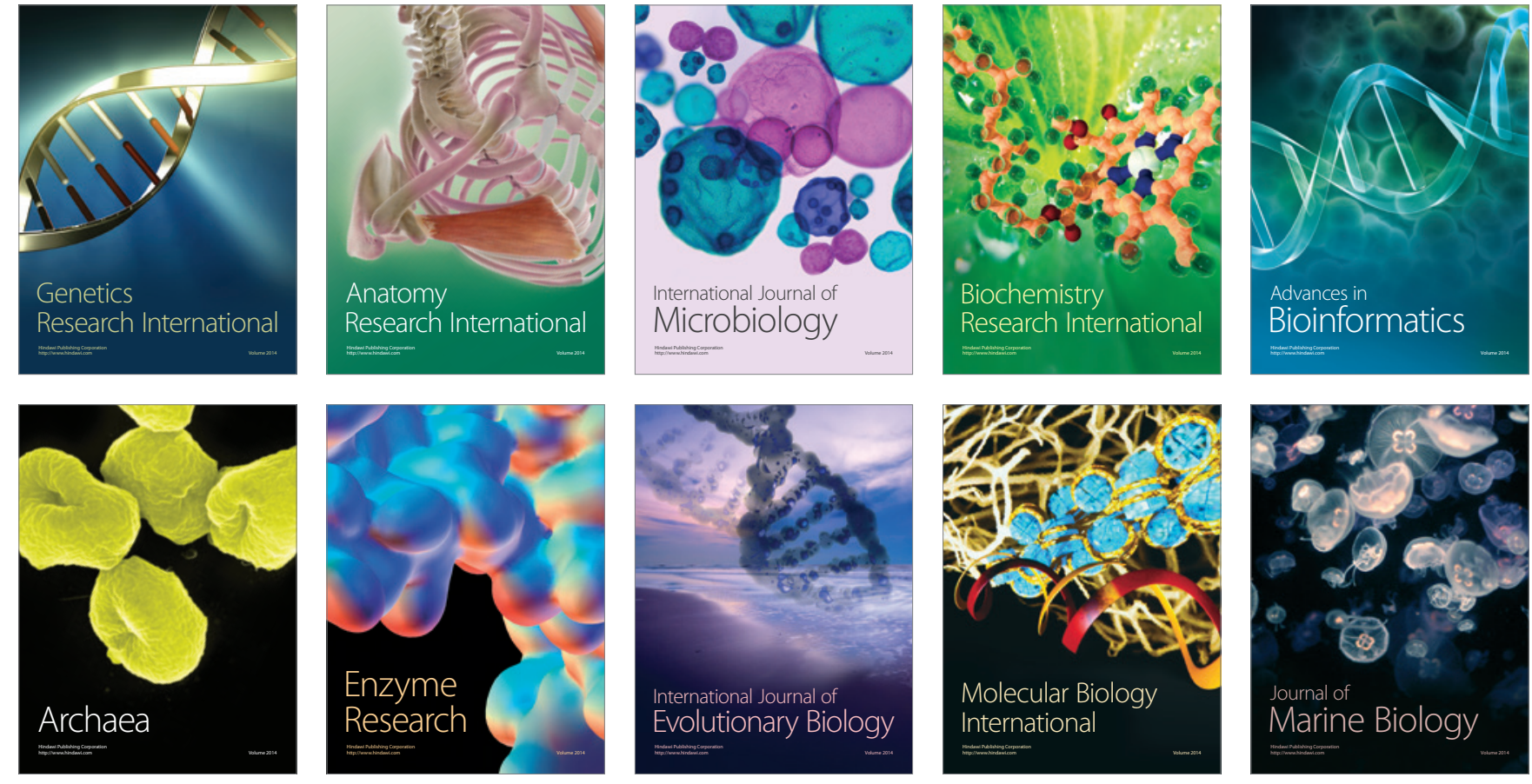